\title{
CARTA SEDIMENTÓLOGICA DA PLATAFORMA CONTINENTAL BRASILEIRA - ÁREA GUAMARÉ A MACAU (NE BRASIL), UTILIZANDO INTEGRAÇÃO DE DADOS GEOLÓGICOS E SENSORIAMENTO REMOTO
}

\author{
Helenice Vital ${ }^{1}$, Iracema Miranda da Silveira ${ }^{1,3}$ e Venerando Eustáquio Amaro ${ }^{1,2}$ \\ Recebido em 06 maio, 2005 / Aceito em 01 novembro, 2005 \\ Received on May 06, 2005 / Accepted on November 01, 2005
}

\begin{abstract}
Studies carried on the Brazilian continental shelf are little developed, especially on the northeastern Brazil, and particularly on Rio Grande do Norte State. This work intends to organize the existent information about the sediment cover on the NE Brazilian continental shelf, and to elaborate a sedimentological chart. The used methodology integrated the sedimentological data with remote sensing processing stored on a georeferenced bank. Ten main facies were mapped on the studied area. Siliciclastic sands are present on shallow waters, near the coast, while carbonate sands are present offshore. The muds are present in the mouth of the rivers and filling channels on the shelf. The sediment cover is one of the most important parameters to understand de shelf system as a whole, because they reflect geological and hydrodynamic processes, present and past, and define, together with others variables, the kind of biological community installed.
\end{abstract}

Keywords: Continental shelf, Marine sediments, Remote sensing, Rio Grande do Norte.

RESUMO. Estudos na plataforma continental brasileira são ainda pouco desenvolvidos, principalmente no Nordeste do Brasil e em particular no Rio Grande do Norte. Visando diminuir esta lacuna este trabalho teve como objetivo reunir as informações existentes sobre a cobertura sedimentar da plataforma brasileira adjacente ao litoral setentrional do RN, com vistas à elaboração de uma carta sedimentológica. A metodologia adotada consistiu na integração de dados sedimentológicos e produtos de sensores remotos armazenados em bancos de dados georreferenciados. Dez fácies sedimentológicas principais foram mapeadas ao longo da plataforma interna setentrional do Rio Grande do Norte, no trecho compreendido entre Guamaré e Macau. 0 fácies areia siliciclástica ocorre em águas rasas ao longo da costa, passando transicionalmente para areais carbonáticas em direção offshore. As lamas estão restritas a foz de rios e ao preenchimento de canyons na plataforma. A cobertura sedimentar constitui-se em um dos parâmetros importantes para a compreensão do ecossistema plataformal como um todo, pois além de refletirem processos geológicos e hidrodinâmicos, passados e atuais, determinam, juntamente com outras variáveis ambientais, os tipos de comunidades biológicas instaladas.

Palavras-chave: plataforma continental, sedimentos marinhos, sensoriamento remoto, Rio Grande do Norte.

\footnotetext{
1 Programa de Pós-graduação em Geodinâmica e Geofísica - PPGG-CCET, Departamento de Geologia, Universidade Federal do Rio Grande do Norte, Campus Universitário, C. P. 1639; 59072-970 Natal, RN, Brasil. Fone: ++55 84 215-3727 (R-13); 215-3808 (R-213); Fax: 215-3683 (R-11) - E-mail: helenice@geologia.ufrn.br 2Departamento de Geologia, Universidade Federal do Rio Grande do Norte; E-mail: amaro@geologia.ufrn.br

${ }^{3}$ Museu Câmara Cascudo, Universidade Federal do Rio Grande do Norte; E-mail: iracema_miranda@hotmail.com.br
} 


\section{INTRODUÇ̃̃o}

A plataforma continental é a extensão do nosso próprio território, e este patrimônio nacional, a chamada "Amazônia Azul", provavelmente com uma quantidade de riquezas muito maior que a "Amazônia verde" tem para nos oferecer, pouco tem sido explorado. Apesar da existência do Programa de Avaliação da Potencialidade Mineral da Plataforma Continental Jurídica Brasileira (REMPLAC), prontificado e aprovado pela Resolução 004/97, de 03/12/97 da Comissão Interministerial para os Recursos do Mar (CIRM, 2000), e da reconhecida importância da Plataforma Continental, poucos estudos tem sido desenvolvidos ao longo da "Amazônia Azul". Essa carência de informações dificulta o estabelecimento de políticas e estratégias governamentais relativas à utilização de recursos naturais (bióticos e abióticos) da plataforma continental (Freire et al., 2002).

Os dados derivados de amostras de sedimentos do fundo marinho são importantes, por exemplo, para: 1) estudar mudanças climáticas visando previsão ambiental; 2) entender o impacto da pesca no habitat bêntico e outras comunidades biológicas; 3) estudar padrões de poluição no mar e quais os mecanismos para ajudar a manter a integridade das áreas costeiras; 4) encontrar fontes de material passível de ser dragado para recomposição de praias; 5) avaliar impactos de deposição de lixo no mar; 6) Iocalizar recursos minerais estratégicos; 7) determinar locais para instalação de cabos submarinos e outras estruturas; 8) fornecer base de dados através de sensores remotos, ajudando a refinar novas técnicas para previsão e caracterização ambiental; 9) entender melhor a Terra e como funciona seu sistema ambiental. A sedimentação na plataforma continental nordeste do Brasil reflete a geologia, clima, drenagem e ambiente tectônico. Devido ao clima tropical e sedimentação terrígena desprezível, esta plataforma é estreita (média de $63 \mathrm{~km}$ de largura) e rasa (na sua maior parte mais rasa que $40 \mathrm{~m}$ ). Em contraste com outras plataformas tropicais, a presença de corais é inexpressiva. Estes são encontrados nos recifes da zona litoral, mas contém apenas alguns dos gêneros construtores de recifes comuns no Oeste das Índias (Laborel, 1967). Os sedimentos terígenos são, na sua grande maioria, relíquias e a sua composição sugere que, no Pleistoceno, 0 clima foi muito similar ao atual (França et al., 1976; Kowsmann \& Costa, 1979; Amaral, 1979; Vital et al., 2005).

A Plataforma Continental Brasileira adjacente ao Estado do Rio Grande do Norte (RN) é uma das áreas menos conhecidas no Brasil. Esta escassez de dados, muito provavelmente, esta relacionada a sua profundidade rasa e a presença de obstáculos proeminentes (recifes), que dificultam a navegação. Os pou- cos estudos desenvolvidos são pontuais e restritos a áreas de exploração de hidrocarbonetos. Pesquisas desenvolvidas mais recentemente na plataforma setentrional do RN foram veiculadas apenas como dissertações, resumos e comunicações de congressos (Costa Neto, 1997; Röber, 2001; Guedes, 2002; Vital et al., 2002a,b,c; Amaro et al., 2002). Assim, este trabalho tem como objetivo reunir as informações existentes sobre a cobertura sedimentar da plataforma brasileira adjacente ao litoral setentrional do RN (Figura 1), com vistas à elaboração de uma carta sedimentológica.

Esta caracterização é importante tanto para analisar as possíveis relações existentes entre a dinâmica e sedimentação do ambiente recente e paleoceanográfico, quanto para análise das situações de risco decorrentes de eventuais derrames de hidrocarbonetos na Plataforma Continental e áreas costeiras adjacentes, tendo em vista a Bacia Potiguar ser o maior produtor de óleo em terra no Brasil, e a exploração no mar ocorrer em águas rasas.

\section{MATERIAIS E MÉTODOS}

A metodologia adotada neste estudo consistiu inicialmente na análise de dados batimétricos (Ministério da Marinha, 1972; Costa Neto, 1997; Röber, 2001; Guedes \& Vital, 2001; Guedes, 2002; Guedes \& Vital, 2002) e amostras existentes no acervo do Laboratório de Geologia e Geofísica Marinha e Monitoramento Ambiental-GGEMMA da Pós-Graduação em Geodinâmica e Geofísica-PPGG/UFRN: 74 amostras coletadas na região de Galinhos e Guamaré em projetos coordenados por H. Vital, em sua maioria usadas anteriormente por Röber (2001) e Guedes (2002) respectivamente em dissertação de mestrado (Cooperação CAU Kiel-UFRN) e Relatório de Graduação em Geologia (DG-UFRN), 44 amostras cedidas do acervo Museu Câmara Cascudo, coletadas entre Guamaré e Macau em projetos coordenados por I. Silveira, e 149 amostras coletadas na região da foz do rio Açu cedidas por L.X. Costa Neto que as utilizou em dissertação de mestrado desenvolvida na UFF (Costa Neto, 1997). Um banco de dados georreferenciados, com acesso para visualização em ArcView 3.2, foi constituído com as amostras selecionadas (amostras com dados incompletos foram descartadas). Com vistas na padronização do mapeamento faciológico, estas amostras inicialmente foram analisadas através do software SAG-Sistema de Análises granulométricas, desenvolvido pela UFF (Dias \& Ferraz, 2004), que classifica os sedimentos de acordo com Shepard (1954), Larsonneur (1977) e Dias (1996). A classificação aqui utilizada foi a de Freire et al. (1997) adaptada de Dias (1996), tendo em vista a predominância de depósitos carbonáticos, formados 


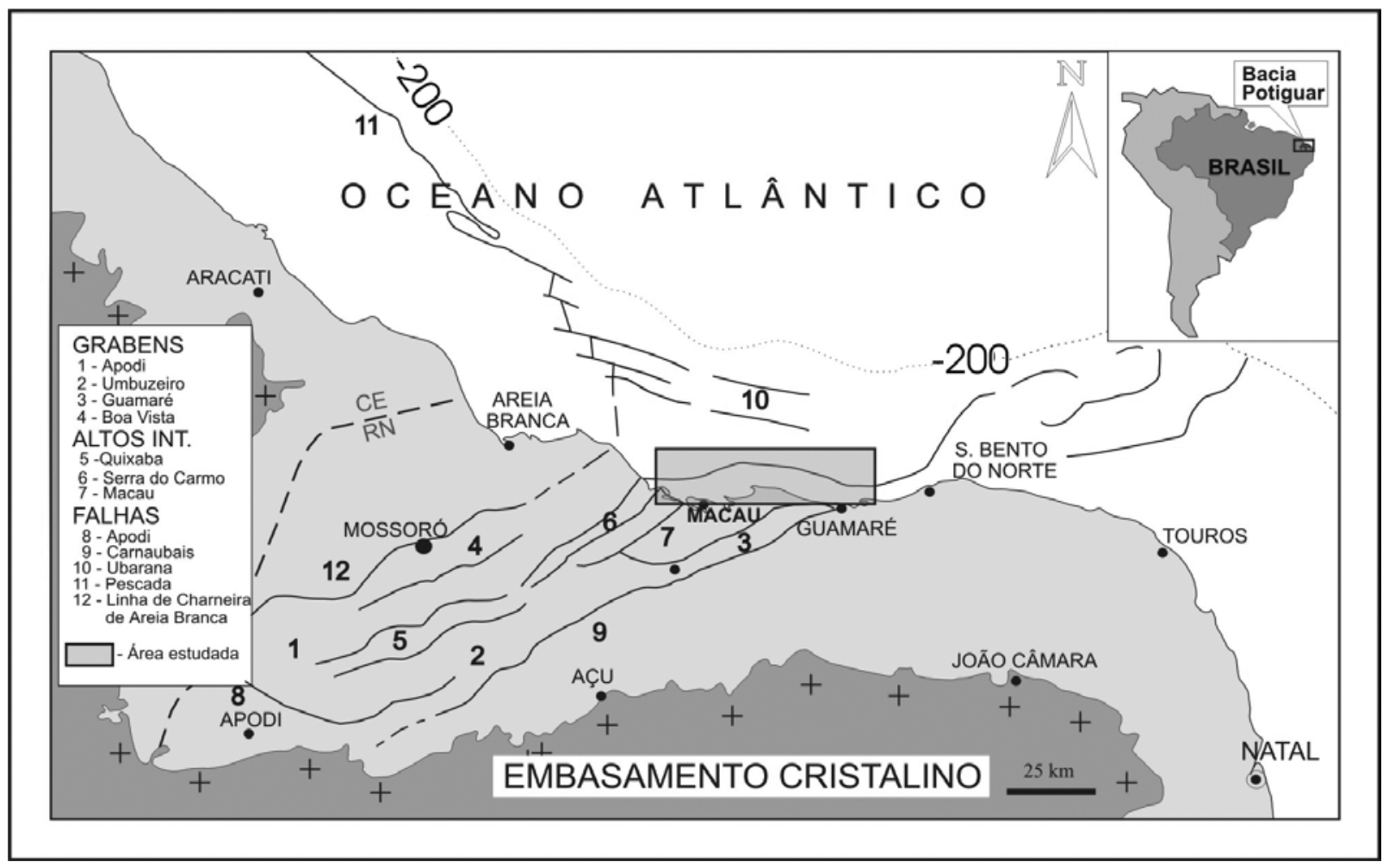

Figura 1 - Arcabouço tectônico da Bacia Potiguar com localização da área em azul. Compilado de Bertani et al. (1990).

Tabela 1 - Classificação de sedimentos do fundo marinho (Freire et al., 1997).

\begin{tabular}{|c|c|c|c|c|}
\hline \multirow{2}{*}{ SUBDIVISÕES PRINCIPAIS } & \multirow{2}{*}{$\begin{array}{l}\text { SEIXOS, GRÂNULOS, } \\
\text { COQUINAS OU RODOLITOS } \\
(\mathrm{L}<15 \% ; \mathrm{Md}>2 \mathrm{~mm})\end{array}$} & \multicolumn{2}{|c|}{$\begin{array}{c}\text { AREIAS } \\
(\mathrm{L}<15 \% \text {; areia+lama }>50 \% ; \mathrm{Md}<2 \mathrm{~mm})\end{array}$} & \multirow{2}{*}{$\begin{array}{c}\text { SEDIMENTOS } \\
\text { LAMOSOS } \\
\quad L>15 \%\end{array}$} \\
\hline & & $15 \%<$ superior a $2 \mathrm{~mm}<50 \%$ & superior a $2 \mathrm{~mm}<15 \%$ & \\
\hline $\begin{array}{c}\text { SEDIMENTO LITOCLÁSTICO } \\
\text { carbonatos }<30 \%\end{array}$ & $\begin{array}{c}\text { CL 1 } \\
\text { cascalho litoclástico }\end{array}$ & $\begin{array}{c}\text { AL1a } \\
\text { areia litoclástica } \\
\text { com grânulos e cascalhos }\end{array}$ & $\begin{array}{c}\mathbf{A L 1 b} \\
\text { areia litoclástica }\end{array}$ & $\begin{array}{c}\text { LL1 } \\
\text { lama terrígena }\end{array}$ \\
\hline $\begin{array}{c}\text { SEDIMENTO LITO-BIOCLÁSTICO } \\
\text { carbonato = } 30 \text { a } 50 \%\end{array}$ & $\begin{array}{c}\text { CL } 2 \\
\text { cascalho lito-bioclástico }\end{array}$ & $\begin{array}{c}\text { AL2a } \\
\text { areia lito-bioclástica } \\
\text { com grânulos e cascalhos }\end{array}$ & $\begin{array}{c}\mathbf{A L 2 b} \\
\text { areia lito-bioclástica }\end{array}$ & $\begin{array}{c}\text { LL2 } \\
\text { marga arenosa }\end{array}$ \\
\hline $\begin{array}{c}\text { SEDIMENTO BIO-LITOCLÁSTICO } \\
\text { carbonatos }=50 \text { a } 70 \%\end{array}$ & $\begin{array}{c}\text { CB1 } \\
\text { cascalho bio-litoclástico }\end{array}$ & $\begin{array}{c}\text { AB1a } \\
\text { areia bio-litoclástica } \\
\text { com grânulos e cascalhos }\end{array}$ & $\begin{array}{c}\mathbf{A B 1 b} \\
\text { areia bio-litoclástica }\end{array}$ & $\begin{array}{c}\text { LB 1 } \\
\text { marga calcária }\end{array}$ \\
\hline $\begin{array}{l}\text { SEDIMENTO BIOCLÁSTICO } \\
\text { carbonato }>70 \%\end{array}$ & $\begin{array}{c}\text { CB2 } \\
\text { cascalho bioclástico }\end{array}$ & $\begin{array}{c}\text { AB2a } \\
\text { areia bioclástica } \\
\text { com grânulos e cascalhos }\end{array}$ & $\begin{array}{c}\text { AB2b } \\
\text { areia bioclástica }\end{array}$ & $\begin{array}{c}\text { LB2 } \\
\text { lama calcária }\end{array}$ \\
\hline
\end{tabular}


por uma mistura biogênica/biodetrítica, constituída por fragmentos de algas calcárias, moluscos, ostracodes, briozoários e foraminíferos que ocorrem apenas nesta região da costa Brasileira (Tabela 1).

Em seguida foi elaborada uma carta base a partir dos dados analisados, na qual as 267 amostras foram plotadas, de acordo com a sua classificação sedimentológica (Tabela 2). Paralelamente, uma cena do satélite LANDSAT-7-ETM+ do ano de 2001 foi recortada às dimensões da área de interesse e processada utilizando-se filtros adaptativos direcionais de modo a ressaltar a morfologia do fundo submarino. Sobre esta imagem foram plotadas as 267 amostras (Figura 2). As amostras foram classificadas em fácies pré-estabelecidas (Tab. 2 e Fig. 2).

Através da integração entre a carta base, análise das amostras selecionadas e a carta obtida a partir da imagem LANDSAT7-ETM+, associada aos dados sonográficos desta área (Vital et al., 2002a, b), foi traçado os limites de fácies e elaborada a carta sedimentológica da área em apreço (Figura 3).

\section{RESULTADOS E DISCUSSÕES}

Tendo em vista o prefixo lito (do grego lithos) significar pedra ou rocha e o termo litoclástico ser comumente utilizado no sentido de clasto sedimentar composto de um tipo de rocha pré-existente (Allaby \& Allaby, 1991), optou-se neste estudo por utilizar o termo siliciclástico, por este ser aplicado a sedimentos que compreendem partículas compostas de fragmentos de rochas e minerais silicáticos, ou seja argilitos, arenitos e conglomerados (Allaby \& Allaby, 1991) e, portanto mais apropriado para a cobertura sedimentar da plataforma continental do Nordeste do Brasil. Os demais critérios da classificação utilizada por Freire et al. (1997) permanecem inalterados. Desta forma os sedimentos foram classificados em 16 fácies conforme Tabela 2. Entretanto apenas as 10 fácies principais foram representadas em mapa (Figura 3).

Por tratar-se de uma plataforma rasa e a cena LANDSAT-7ETM+ utilizada ter sido obtida em período de águas excepcionalmente límpidas (baixo volume de sedimentos em suspensão) e ausência de nuvens foi possível visualizar o fundo marinho até a profundidade de $25 \mathrm{~m}$. A imagem multiespectral LANDSAT 7-ETM+ integrada em sistema de cores IHS (Intensity-HueSaturation) e submetida à filtragem direcional permitiu reconhecer diferentes feições submersas de grande escala (Figura 2) com base na cor clara (áreas mais rasas) versus escura (áreas mais profundas), tais como: 1) fundo escuro homogêneo em águas mais rasas que 10 metros, associado a fundo plano; 2) Campos de dunas Iongitudinais subaquosas muito grandes (sentido de Ash- ley, 1990) são encontrados entre 1,5 e 12 quilômetros offshore. São em geral assimétricas e apresentam entre 2 a 3,5 m de altura (Röber, 2001). Próximo à costa, as dunas subaquosas Iongitudinais são aproximadamente lineares orientadas na direção ENEWSW $\left(250^{\circ}\right)$ com cerca de 12 quilômetros de comprimento. Mais distante da costa em direção offshore as dunas subaquosas apresentam extensão de mais de 20 quilômetros, estando separadas uma das outras por uma distância mais ou menos uniforme de 1 a 1.5 quilômetros e com 300 a 500 metros de largura (Röber, 2001). As dunas subaquosas longitudinais podem ser subdividas em bem visíveis a leste (em frente a Guamaré-Galinhos) e menos visíveis a oeste (direção a Macau); 3) campos de dunas transversais muito grandes. Apresentam orientação NE-SW, extensão da ordem de 5 a 10 km, 300 a $400 \mathrm{~m}$ de largura e altura média de $3 \mathrm{~m}$. São observadas a profundidades maiores que $10 \mathrm{~m}$; 4) recifes, linha continua offshore bordejando as dunas transversais e separando-as de área mais profundas sem penetração dos sensores remotos; 5) vale inciso (antigo canyon do rio Açu), em frente a Macau, marcado por uma margem leste relativamente íngreme e pronunciada, e uma margem oeste com apenas um declive suave, (Schwarzer et al., no prelo); e 6) quebra da plataforma, marcada pela ausência de penetração dos sensores remotos. A integração entre 0 tipo de sedimento e a morfologia do fundo marinho associado permitiu uma melhor delimitação dos fácies sedimentológicas que compõem a cobertura sedimentar da plataforma.

\section{Caracterização Sedimentológica}

A distribuição de fácies sedimentológicas ao longo da plataforma interna setentrional do Rio Grande do Norte, no trecho compreendido entre Guamaré e Macau, permitiu o reconhecimento de 16 fácies distintas, entretanto apenas as 10 principais são representadas na Figura 3.

A fácies areia siliciclástica ocorre ao longo da costa, na região entre Guamaré e foz do rio Açu (Macau), em áreas de fundo submarino onde as formas de fundo são bem desenvolvidas, com uma boa visibilidade e representadas pelas dunas Iongitudinais com 2 a 3,5 $\mathrm{m}$ de altura. As dunas longitudinais bem visíveis tendem a concentrar areia siliciclástica nas porções mais altas (crista); enquanto areias silici-bioclásticas com grânulo e cascalho e areias biosiliciclásticas com grânulo e cascalho concentram-se nas partes mais profundas entre as dunas de areia (calhas). Entretanto, devido à escala utilizada na carta sedimentológica da Figura 3 estas três fácies não foram diferenciadas, sendo representadas apenas pela fácies areia siliciclástica. Os sedimentos constituintes desta fácies apresentam granulome- 


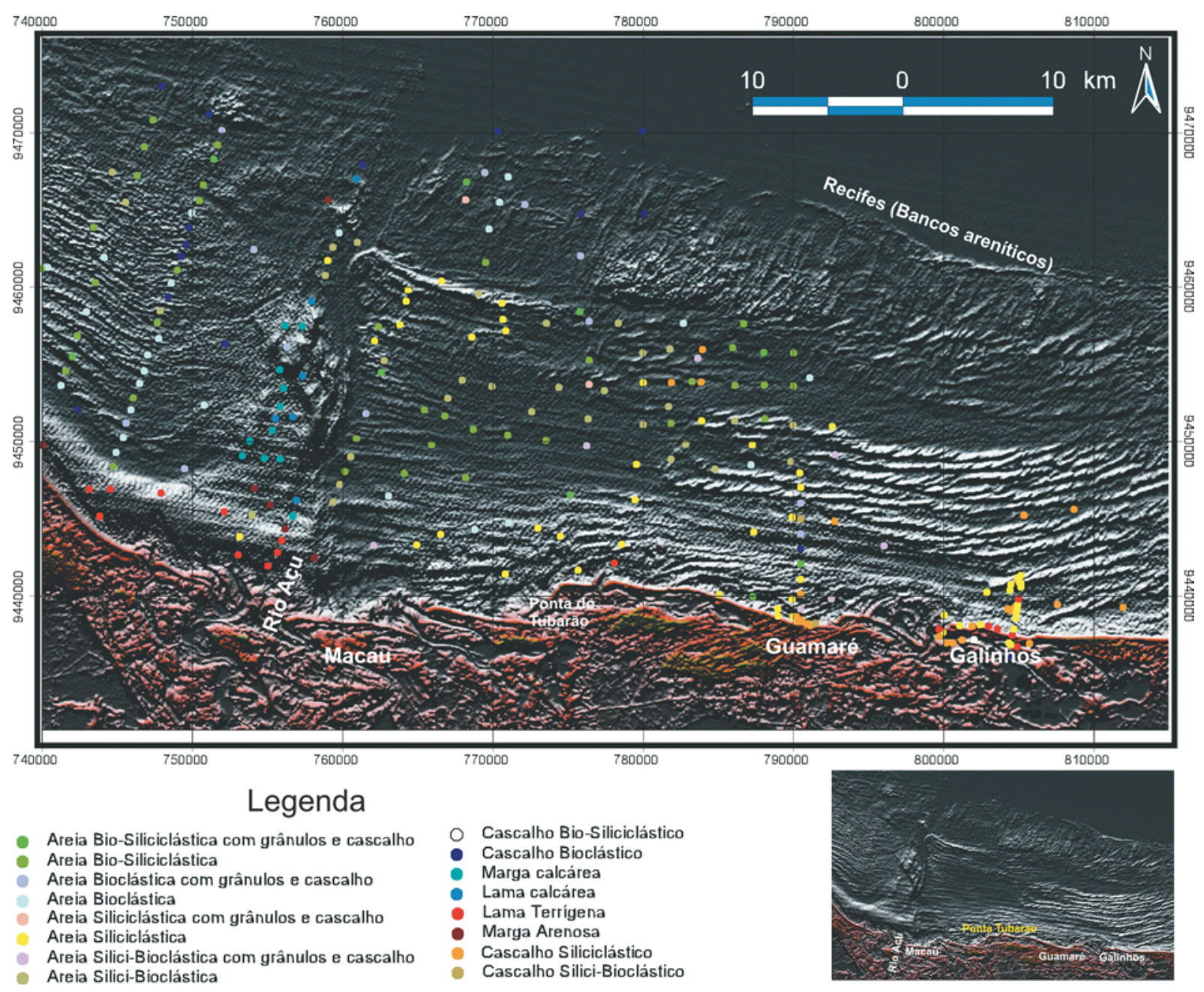

Figura 2 - Amostras de sedimentos analisadas plotadas sobre imagem Landsat ressaltando o fundo marinho. Cada cor corresponde a um fácies, conforme Tabela 2. Continente em tons de preto e rosa; Plataforma em tons de preto e branco.

tria variando de areia média a grossa, os grãos são moderadamente a pobremente selecionados, variando de subangulosos a arredondados com grau de esfericidade predominando nas classes esférica a muito esférica.

A fácies lama terrígena está limitada às áreas próximas a desembocadura dos rios e / ou braços de mar existentes na região, sendo mais proeminente na porção a $\mathrm{E}$ do rio $\mathrm{Açu}$, cujos sedimentos em suspensão são levados pela deriva litorânea ao atingirem o mar. A fácies marga arenosa, lama calcária e marga calcária ocorrem preenchendo o canyon submerso, resquício do antigo vale do rio Açu, aumentando o conteúdo de carbonato à medida que se distancia da costa. A margem leste do canal é relativamente íngreme e pronunciada, tendendo a concentrar lama calcária, enquanto a margem oeste consiste apenas de um declive suave onde há uma maior concentração de marga calcária.

A fácies areia biosiliciclástica ocorre de forma aproximadamente elíptica, na região offshore entre o a foz do rio Açu e a Ponta do Tubarão em fundo plano. Esta fácies ocorre ainda na forma de uma faixa contínua offshore acompanhando a isóbata de 10 metros, após o término das areias siliciclásticas. Neste caso, as formas de fundo apresentam menor dimensão que as desenvolvidas nas areias siliciclásticas e estão orientados preferencialmente na direção NE-SW (dunas transversais), evidenciando diferentes regimes hidrodinâmicos.

A fácies areia silicibioclástica ocorre preferencialmente offshore, circundando a fácies areia biosiliciclástica, próximo à foz do rio Açu, apresentando formas de fundo menos desenvolvi- 
Tabela 2 - Classificação modificada de Freire et al. (1997) para amostras de fundo da Plataforma Continental do RN: Guamaré a Macau.

\begin{tabular}{|c|c|c|}
\hline $\begin{array}{l}\text { NÚMERO } \\
\text { DA FÁCIES }\end{array}$ & $\begin{array}{l}\text { NÚMERO DE } \\
\text { AMOSTRAS }\end{array}$ & CLASSIFICAÇÃO DOS SEDIMENTOS \\
\hline 1 & 10 & $\begin{array}{l}\text { Areia Bio-Siliciclástica com grânulos e cascalho }- \text { AB1a } \\
\text { (C=50 a 70\%; } \mathrm{L}<15 \% \text {; areia+lama }>50 \% \mathrm{Md}<2 \mathrm{~mm} \text {; } \\
15 \%<\text { superior a } 2 \mathrm{~mm}<50 \% \text { ) }\end{array}$ \\
\hline 2 & 37 & $\begin{array}{l}\text { Areia Bio-Siliciclástica - AB1b } \\
\text { (C=50 a 70\%; } \mathrm{L}<15 \% \text {; areia+lama }>50 \% \mathrm{Md}<2 \mathrm{~mm} \text {; } \\
\text { superior a } 2 \mathrm{~mm}<15 \% \text { ) }\end{array}$ \\
\hline 3 & 12 & $\begin{array}{l}\text { Areia Bioclástica com grânulos e cascalho }- \text { AB2a } \\
(C>70 \% ; \mathrm{L}<15 \% \text {; areia+lama }>50 \% \mathrm{Md}<2 \mathrm{~mm} \text {; } \\
15 \%<\text { superior a } 2 \mathrm{~mm}<50 \%)\end{array}$ \\
\hline 4 & 27 & $\begin{array}{l}\text { Areia Bioclástica - AB2b } \\
(\mathrm{C}>70 \% \text {; } \mathrm{L}<15 \% \text {; areia+lama }>50 \% \mathrm{Md}<2 \mathrm{~mm} \text {; } \\
\text { superior a } 2 \mathrm{~mm}<15 \%)\end{array}$ \\
\hline 5 & 2 & $\begin{array}{l}\text { Areia Siliciclástica com grânulos e cascalho - AS1a } \\
(\mathrm{C}<30 \% \text {; L }<15 \% \text {; areia+lama }>50 \% \mathrm{Md}<2 \mathrm{~mm} \text {; } \\
15 \%<\text { superior a } 2 \mathrm{~mm}<50 \%)\end{array}$ \\
\hline 6 & 58 & $\begin{array}{l}\text { Areia Siliciclástica - AS1b } \\
(\mathrm{C}<30 \% \text {; } \mathrm{L}<15 \% \text {; areia+lama }>50 \% \mathrm{Md}<2 \mathrm{~mm} \text {; } \\
\text { superior a } 2 \mathrm{~mm}<15 \%)\end{array}$ \\
\hline 7 & 7 & $\begin{array}{l}\text { Areia Silici-Bioclástica com grânulos e cascalho }- \text { AS2a } \\
\text { (C=30 a 50\%; } L<15 \% \text {; areia+lama }>50 \% \mathrm{Md}<2 \mathrm{~mm} \text {; } \\
15 \%<\text { superior a } 2 \mathrm{~mm}<50 \% \text { ) }\end{array}$ \\
\hline 8 & 31 & $\begin{array}{l}\text { Areia Silici-Bioclástica- AS2b } \\
\text { (C=30 a } 50 \% ; L<15 \% \text {; areia+lama }>50 \% M d<2 \mathrm{~mm} \text {; } \\
\text { superior a } 2 \mathrm{~mm}<15 \% \text { ) }\end{array}$ \\
\hline 9 & 1 & $\begin{array}{l}\text { Cascalho Bio-Siliciclástico - CB1 } \\
\text { (C=50 a } 70 \% ; L<15 \% ; M d>2 m m)\end{array}$ \\
\hline 10 & 13 & Cascalho Bioclástico - CB2 (C>70\%;L<15\%; Md >2mm) \\
\hline 11 & 11 & Marga calcária - LB1 (C=50-70\%; L >15\%) \\
\hline 12 & 6 & Lama Calcária - LB2 (C > 70\%; L > 15\%) \\
\hline 13 & 17 & Lama Terrígena - LL1 (C<30\%; L>15\%) \\
\hline 14 & 6 & Marga Arenosa - LL2 (C=30-50\%; L>15\%) \\
\hline 15 & 27 & Cascalho Siliciclástico - CS1 (C <30\%; L <15\%; Md >2mm) \\
\hline 16 & 2 & Cascalho Silicibioclástico - CS2 (C=30-50\%; L <15\%; Md >2mm) \\
\hline
\end{tabular}

Abreviatura: $\mathrm{C}=$ carbonato; $\mathrm{L}=$ lama; $\mathrm{Md}=$ mediana

das (menos visíveis) que a fácies areia siliciclástica, embora com a mesma direção. Provavelmente, estas formas de fundo são menos visíveis na imagem de satélite devido à presença dos carbonatos que não apresentam reflexão tão intensa quanto às areias siliciclásticas constituídas predominantemente de quartzo.

As areias bioclásticas ocorrem preferencialmente como uma faixa contínua após a areia biosiliciclástica, diferenciando-se da mesma apenas pela maior profundidade; ocorrem ainda após a foz do rio Açu, onde desenvolvem formas de fundo aproximadamente paralelas a costa (Dunas longitudinais). Estas formas de fundo apresentam entre 2 a 1,5 metros de altura e tendem a concentrar areia bioclástica nas porções mais altas (crista); enquanto areias bioclásticas com grânulo e cascalhos concentramse nas partes mais profundas entre as formas de fundo. Assim, na carta sedimentológica estas duas fácies são indistintas, sendo representadas apenas pela fácies areia bioclástica. 


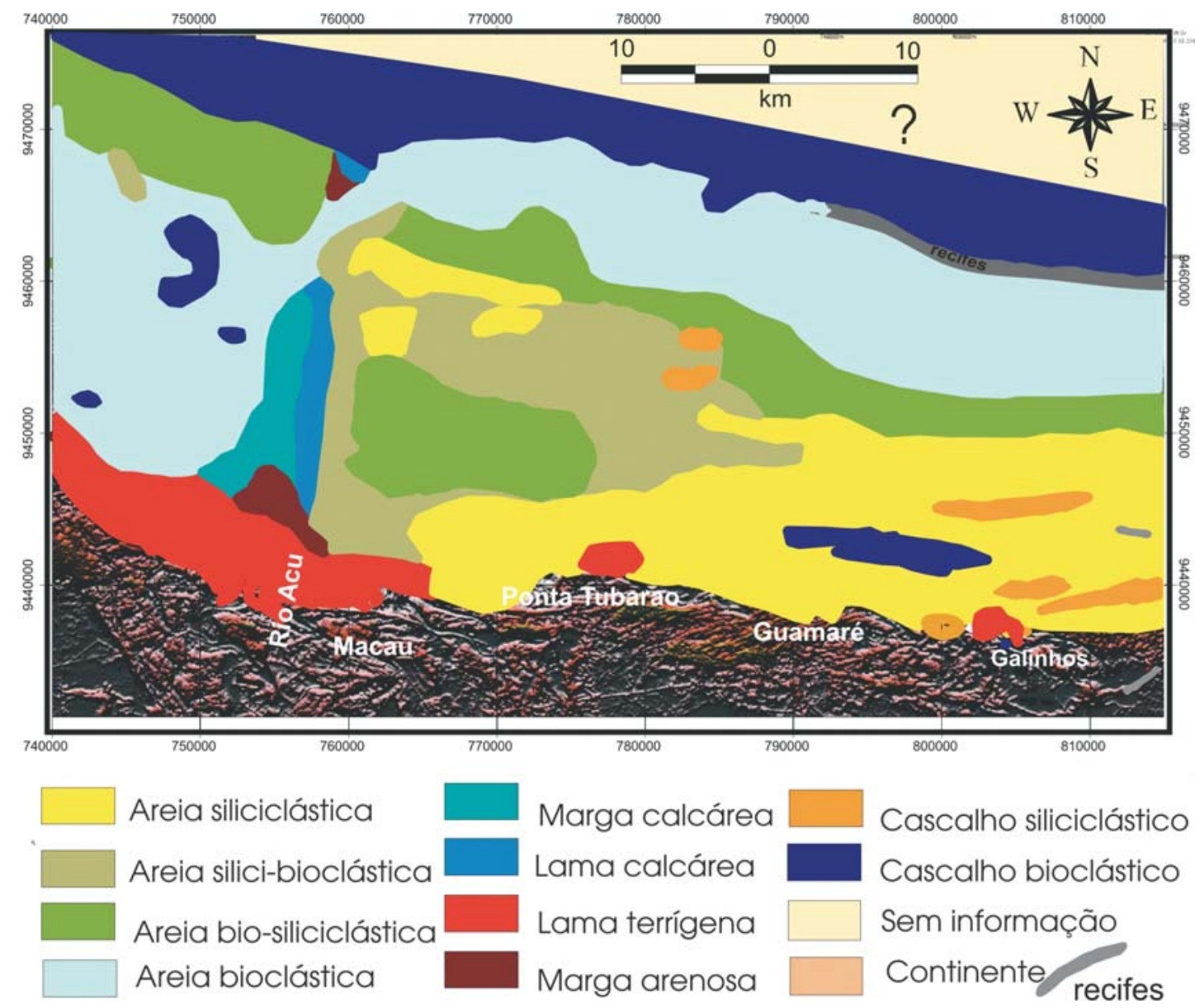

Figura 3 - Carta sedimentológica para a plataforma continental brasileira entre Guamaré e Macau (NE Brasil).

0 cascalho bioclástico ocorre preferencialmente em profundidades maiores de 25 metros, acompanhando esta isóbata. 0 cascalho siliciclástico e em menor proporção o cascalho bioclástico, ocorre na forma de bolsões em áreas mais rasas, onde o fundo submarino é plano. Neste último caso poderiam estar associados a depósitos residuais formados por correntes de marés de alta velocidade. Embora estudos hidrodinâmicos sistemáticos sejam ausentes nesta região, dados recentes registram localmente velocidades de correntes da ordem de $100 \mathrm{~cm} / \mathrm{s}$. Os cascalhos do fundo provavelmente são relíquiares de uma superfície erosional mais antiga que foi formada durante nível de mar baixo no Pleistoceno.

\section{CONCLUSÕES}

A integração de dados de sensores remotos e dados sedimentológicos mostrou-se apropriada para geração de cartas sedimentológicas em áreas submersas rasas e de difícil acesso (e.g. presença de obstáculos) onde a presença de rios com grande aporte sedimentar é desprezível, como no Nordeste do Brasil. Os resultados obtidos neste trabalho para o litoral setentrional do Rio Grande do Norte permitiram, a partir do processamento digital de imagens, a delimitação mais precisa do padrão da distribuição sedimentar no fundo marinho, tendo em vista as diferentes respostas espectrais em função da composição e textura da cobertura sedimentar, que por sua vez esta relacionada à morfologia do fundo marinho.

0 tipo de informação aqui obtido, associado com outros parâmetros ambientais, irá subsidiar na determinação dos tipos de comunidades bióticas associadas, bem como uma melhor compreensão dos ambientes deposicionais modernos.

\section{AGRADECIMENTOS}

Esta pesquisa foi financiada com recursos CNPq (Grant PQ Proc. 3508811999-5) e Projetos PETRORISCO (REDE05/FINEP/ 
CTPETRO/PETROBRAS/CNPq), CNPq (Proc. 500407/2004-5) e MAR-RN (FINEP/CTINFRA). Os autores agradecem ainda a UFRN (DG, PPGG, CCET) pela disponibilização da infra-estrutura necessária ao desenvolvimento da pesquisa, ao CNPq pela concessão de bolsa produtividade de pesquisa ao primeiro autor e aos revisores anônimos, que colaboraram para melhorar a qualidade deste artigo. Instituição participante do Programa de Geologia e Geofísica Marinha-PGGM.

\section{REFERÊNCIAS}

ALLABY A \& ALLABY M. 1991. The Concise Oxford Dictionary of Earth Sciences. Oxford University press. 410p.

AMARAL CAB. 1979. Recursos Minerais da Margem Continental Brasileira e das Áreas Oceânicas Adjacentes. Projeto REMAC. Vol.10. Petrobras. Rio de Janeiro. $112 \mathrm{p}$.

AMARO VE, VITAL H, ALVES AL, LIMA ZMC, TABOSA WF, CALDAS LHO \& SOUTO MVS. 2002. Application of Remote Sensing Multitemporal/Multisensor Data Analysis and GIS Database for Coastline change monitoring and nearshore morphology detection in RN State, NE Brazil. Ocean Sciences Meeting, American Geophysical Union, Honolulu-Hawaii and EOS Transaction, American Geophysical Union, 83(4): p. OS92.

ASHLEY GM. 1990. Classification of Large-Scale Subaqueous Bedforms: A New Look at an Old Problem. Journal of Sedimentary Petrology, 60: 160-172.

BERTANI RT, COSTA ID \& MATOS RMD. 1990. Evolução tectosedimentar, estilo estrutural e habitat do petróleo na Bacia Potiguar. In: GABAGLIA GPR \& MILANI EJ (Coord.). Origem e Evolução das Bacias Sedimentares. Rio de Janeiro: PETROBRAS. p. 291-310.

CIRM. Comissão Interministerial para os Recursos do Mar. 2000. 0 Modelo Brasileiro para o desenvolvimento das atividades voltadas para os Recursos do Mar. Boletim SECIRM, julho.

COSTA NETO LX. 1997. Evolução geológica-geomorfológica recente da plataforma continental interna ao largo do delta do Rio Açu, Macau - RN. Programa de pós-graduação em geologia e geofísica marinha da Universidade Federal Fluminense, Rio de Janeiro, Dissertação de Mestrado, 214 pp.

DIAS GTM. 1996. Classificação de sedimentos marinhos, proposta de representação em cartas sedimentológicas. In: Congresso Brasileiro de Geologia, 39; Salvador. Anais... Salvador: SBG, 1996, 3: 423-426.

DIAS GTM \& FERRAZ CB. 2004. SAG - Sistema de Análise GranuIométrica. Manual do Usuário. Publicação interna Dept. GeologiaLagemar/UFF. Disponível em: 〈http://www.igeo.uff.br〉.

FRANÇA AMC, COUTINHO PN \& SUMMERHAYES CP. 1976. Sedimentos superficiais da margem continental nordeste brasileira. Revista Brasileira de Geociências, 6: 147-164.
FREIRE GSS, CAVALCANTI VMM, MAIA LP \& LIMA SF. 1997. Classificação dos Sedimentos da Plataforma Continental do Estado do Ceará. In: Simpósio de Geologia do Nordeste; Fortaleza. Anais... Fortaleza, 1997, p. 209-211.

FREIRE GSS, FILHO WFS, GOMES DF, LIMA SF \& MONTEIRO LHU. 2002. Províncias sedimentares da Plataforma Continental do nordeste do Brasil. In: Congresso Brasileiro de Geologia, 41; João Pessoa. Anais.. João Pessoa: SBG, 2002, p. 90.

GUEDES IMG. 2002. Mapeamento da área de influência dos dutos de Gás e Óleo do Pólo de Guamaré (RN). Relatório de Graduação do Curso de Geologia da Universidade Federal do Rio Grande do Norte-UFRN. $78 \mathrm{pp}+$ anexos.

GUEDES IMG \& VITAL H. 2001. Caracterização da Plataforma interna adjacente a área de influência dos dutos de gás e óleo do pólo de Guamaré-RN. In: Congresso da Associação Brasileira de Geologia do Quaternário-ABEQUA, VIII; Imbé. Anais... Imbé, 2001, p. 529-531.

GUEDES IMG \& VITAL H. 2002. Mapeamento da área de dutos do pólo petrolífero de Guamaré-RN. In: Congresso Brasileiro de Geologia, 41; João Pessoa. Anais... João Pessoa: SBG, 2002.

KOWSMANN RO \& COSTA MPA. 1979. Sedimentação Quaternária da Margem Continental Brasileira e das Áreas Oceânicas Adjacentes. Projeto REMAC, 8: 55.

LABOREL JL. 1967. A revised list of Brazilian scleractinian corals and description of a new species. Postilla, Peabody Mus. Nat. Hist. Yale Univ., 107: 14 pp.

LARSONNEUR C. 1977. La cartographie des dépôt meubles sur le plateau continental français: méthode mise au point et utilisée en Manche. J. Rech. Oceanogr. 2: 34-39.

MINISTÉRIO DA MARINHA. 1972. Diretoria de Hidrografia e Navegação da Marinha do Brasil. Carta Náutica da DHN n 700, Escala 1:316.220. $2^{\mathrm{a}}$ Edição

RÖBER V. 2001. Structure and dynamics of the inner shelf north of Galinhos, Rio Grande do Norte (NE - Brazil). Institute of Geosciences, University Christian Albrechts zu Kiel, Germany. Tese, 76 pp.

SHEPARD FP. 1954. Nomenclature based on sand-silt-clay ratios. J. Sediment. Petrol. 24(3): 151-158.

SCHWARZER K, STATTEGGER K, VITAL H \& BECKER M. No prelo. Holocene Coastal Evolution of the Rio Açu Area (Rio Grande do Norte, Brazil). Journal of Coastal Research, SI 39 (Proceedings of The $8^{\text {th }}$ International Coastal Symposium), Itajaí, SC - Brazil.

VITAL H, AMARO V, TABOSA WFT, GUEDES IMG, STATTEGGER K \& CALDAS LHO. 2002a. Pattern of sediment distribution in setentrional coast of RN State, NE Brazil. Ocean Sciences Meeting, American Geophysical Union, Honolulu-Hawaii, 2002 and EOS Transaction, American Geophysical Union, 83(4): p. OS16 
VITAL H, AMARO VE, STATTEGGER K, SCHWARZER K, TABOSA W, FRAZÃO EP, SILVEIRA IM \& CALDAS LHO. 2002b. Interactions of sea level and tectonics on large scale bedforms preserved on a tropical shelf: The Rio Grande do Norte shelf, NE Brazil. IGCP464. Continental shelves during Last Glacial Cycle. $2^{\text {nd }}$ Conference Abstracts volume, p. 99.

VITAL H, AMARO VE, SILVEIRA IM, STATTEGGER K, ALVES AL, CALDAS LHO, GUEDES IMG, TABOSA WFT \& SOUSA FE. 2002c. Distribuição de Sedimentos na Região Costeira e Plataforma Continental Setentrional do
Estado do Rio Grande do Norte. In: Congresso Brasileiro de Geologia, 41; João Pessoa. Anais... João Pessoa: SBG, 2002, p. 120.

VITAL H; ESTEVES LS; ARAÚJO TCM \& PATCHNEELAM SM. 2005. Geologia e Geofísica da Plataforma Continental Brasileira. In: SOUZA CRG, SUGUIO K, OLIVEIRA AMS \& OLIVEITA PE. (Eds). "Quaternário do Brasil”. Holos Editora, Ribeirão Preto. Associação Brasileira de Estudos do Quaternário-ABEQUA, p. 153-175.

\section{NOTAS SOBRE OS AUTORES}

Helenice Vital. Graduada em Geologia pela Universidade Federal do Rio Grande do Norte e Mestre em Geologia Marinha e Ambiental pela Universidade Federal do Pará. Concluiu seu doutorado em 1996 na Christian Albrechts Universität zu Kiel, Alemanha e desde 1997 tem desenvolvido pesquisas na área de Geologia e Geofísica Marinha na Universidade Federal do Rio Grande do Norte. É pesquisadora do CNPq desde 1999.

Iracema Miranda da Silveira. Graduada em Geografia e mestre em Geodinâmica pela Universidade Federal do Rio Grande do Norte. Vem desenvolvendo pesquisas e trabalhos na área de Erosão Costeira e Geomorfologia Marinha como professor e pesquisador da UFRN desde 1979.

Venerando Eustáquio Amaro. Graduado em Geologia pela Universidade Federal do Mato Grosso e Mestre em Geologia Econômica pela Universidade de Brasília. Desenvolveu pesquisas durante dois anos na Université Claude Bernard-Lyon I, França como parte de doutorado em Sensoriamento Remoto concluído em 1998 na USP; vem trabalhando na área de Geoprocessamento e Sensoriamento Remoto na Universidade Federal do Rio Grande do Norte desde 1989. 